\title{
Non-isothermal experimental and simulation study of residual wall thickness in gas assisted injection moulding
}

\author{
P. Olley*, L. Mulvaney-Johnson and P. D. Coates
}

\begin{abstract}
A methodical 'design of experiment' approach is used to assess the effect of key control parameters on residual wall thickness (RWT) in gas assisted injection moulding. An empirical model is produced from which the experimental RWT can be determined at any interpolated point. This model includes only those terms with proven statistical significance. The 'true' thermal boundary conditions are determined for a 1-D approximation to the system; this is sufficient to determine the error in a simulation method that enforces coolant temperature as the mould boundary condition for temperature. It is shown that errors in heat flux and wall temperature are small. A 3-D finite element, pseudo-concentration implementation is presented, with a novel method for simulation of internal gas injection. The simulation is shown to give good agreement with the experimental rate of growth of wall thickness as gas delay is increased; good qualitative agreement is shown for other control parameters.
\end{abstract}

Keywords: Gas assisted injection moulding, Residual wall thickness, Experimental model, Heat flow, Simulation

\section{Introduction and background}

Gas assisted injection moulding (GAIM) is a relatively new process to produce plastic parts with hollow sections. ${ }^{1}$ In the method, molten polymer, or liquid rubber, is injected to partly fill a mould; gas is then injected under pressure to hollow out the melt, leaving a residual solid wall. ${ }^{2}$ Advantages over conventional injection moulding are many-fold: less polymer is used and lower melt pressures are needed allowing smaller and lighter equipment. Less material gives quicker cooling, and an increase in production rate. The finished products have lower residual stress and warpage, and the effects of shrinkage on surface finish can be minimised by maintaining a packing gas-pressure. For these advantages, the complexities of control are increased; gas pressure, temperature and timing play key roles in determining gas penetration and control of fingering. ${ }^{3}$ The objective mechanical properties of parts produced by GAIM have been examined. Chien et al. found little change in tensile strength and yield stress for polypropylene (PP), or polystyrene (PS). ${ }^{4}$ Also bending properties were linear with moment of inertia suggesting little change in material properties when processed by GAIM. Nylon was found to be more complex with process parameters affecting the degree of crystallinity, which affected all measured properties.

School of Engineering Design and Technology/IRC in Polymer Engineering, Chesham Building, University of Bradford, Bradford BD7 1DP, UK

*Corresponding author, email p.olley@bradford.ac.uk
The capability of GAIM simulation has increased steadily since Khayat et al. presented a method for the simulation of gas penetration (using the boundary element method). ${ }^{5}$ There have been a number of HeleShaw flow formulations, whereby 3-D problems are first simplified to 2-D or 1-D 'equivalent' sections; a recent model extends to shrinkage and secondary flows. 6,7 Haagh et al. introduced a pseudo-concentration method, using finite elements, for tracking gas penetration in planar, axisymmetric and 3-D objects. ${ }^{8}$ The method is computationally expensive, but simulates aspects of the physics of the process. The method was advanced by non-isothermal simulation, and the use of the CrossWLF model to give shear thinning and temperaturedependent viscosity. ${ }^{9,10}$ For the range covered, it was found that in both experiment and simulation, shear thinning causes a reduction in residual wall thickness, whereas non-isothermal simulation (with cooling from walls) gives an increase in wall thickness. ${ }^{11}$ Ilinca and Hetu used a two-variable pseudo-concentration method to differentiate between the propellant gas, and air initially in a mould. ${ }^{3}$ This allowed the significantly different properties of air, and high pressure gas to be incorporated. Dimakopoulos and Tsamopoulos studied gas penetration when viscoelasticity was included in the simulation model using a Phan Thien-Tanner (PTT) and a Boger constitutive model. ${ }^{12} \mathrm{~A}$ moving mesh method was employed, and isothermal conditions were used. The wall thickness was found to increase greatly with elasticity, particularly for the Boger fluid with up to $60 \%$ wall thickness; the PTT model gave slightly lower wall thicknesses, and the difference was ascribed to the shearthinning of the PTT model. Polynkin and coworkers 


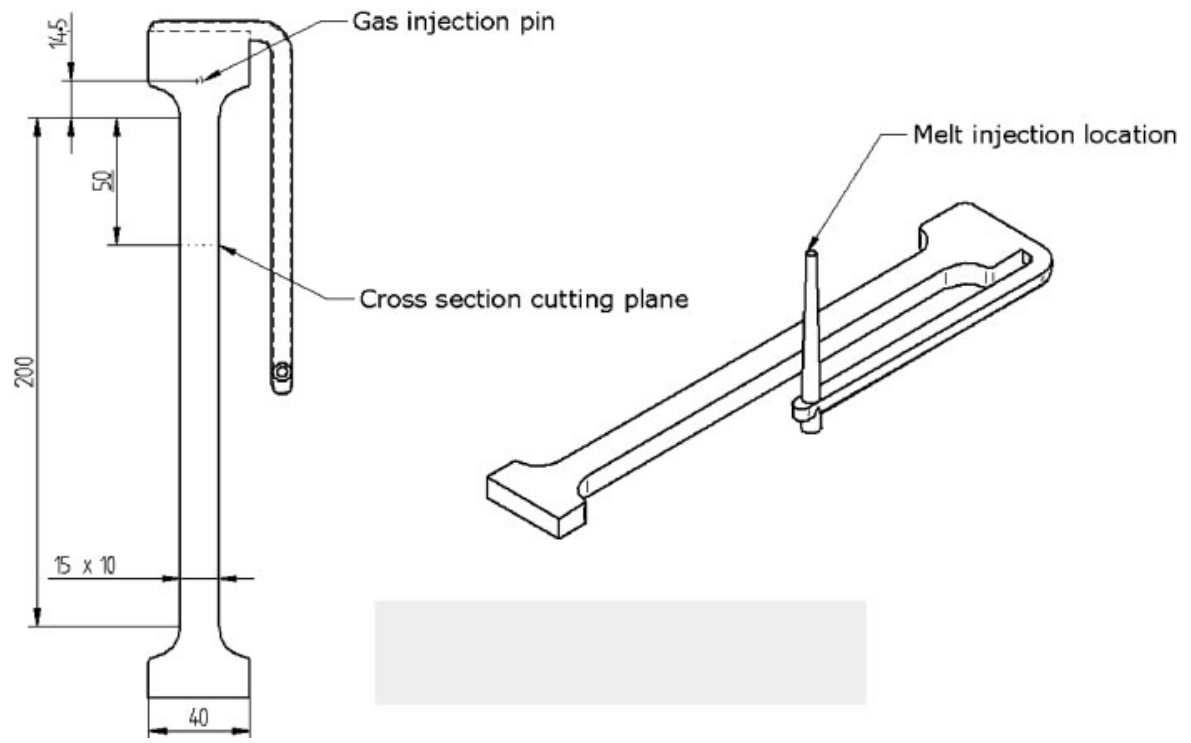

1 Geometry of mould, with dimensions in $\mathrm{mm}$; radii of testpiece shoulders were $15 \mathrm{~mm}$; cross-section used for measurements of residual wall thicknesses is shown

quantified the effects on wall thickness of a number of significant dimensionless parameters for circular tubes, ${ }^{13,14}$ and for non-circular tubes. ${ }^{15}$ The studies included the effects of momentum (Reynold's number), and thermal energy (Biot number, Pearson number and Fourier number). Shrinkage during cooling, and subsequent secondary flows have been incorporated into a pseudo-concentration model. ${ }^{16,17}$ Time-dependent heat transfer coefficients were computed for an entire mould (assuming only perfect thermal contact between polymer and mould surface, and a fixed coolant-mould heat transfer coefficient). Initially, an averaged heat transfer coefficient was calculated and applied during simulation of a 3-D product, ${ }^{16}$ and local variation of the heat transfer coefficient was later used. ${ }^{17,18}$

In the present study, a methodical 'design of experiment' approach was used to assess the effect of key control parameters on residual wall thickness. The control parameters that are demonstrably significant were identified by statistical tests, and an experimentally based model was produced from which the experimental residual wall thickness could be determined at any interpolated point. This model included only those terms with proven statistical significance.

A study was made on a 1-D characteristic system to determine the error in the simulation method of enforcing coolant temperature as the mould boundary condition for temperature. It was shown that errors in heat flux and mould/polymer interface temperature are small using this method, much smaller than discretisation errors from the mesh $(0.2 \mathrm{~mm}$ mesh over a $5 \mathrm{~mm}$ half-width). A 3-D finite element, pseudo-concentration model is presented, with a novel method for simulation of gas injection at an internal point; this replicates injection from an internal point through a hollow needle, as used in industrial GAIM processes. The simulation is shown to give good agreement with the rate of growth of wall thickness as gas delay is increased; good qualitative agreement is shown for injection velocity, gas pressure, initial melt temperature, and tool temperature variation over the range covered by experiment. The omission of viscoelasticity in the method is identified as the most significant simplification to the real system.

\section{Experimental}

The experimental studies were carried out on an industrial scale injection moulding machine (IMM) fitted with a gas assist control system. The IMM was a Battenfeld CDK 750 with UNILOG 9000 control; the gas assist unit was an 'Airmould' pressure generation unit with separate control system. The polymer used in the study was BP HDPE 5050EA.

The mould geometry was that of a tensile test specimen, shown in detail in Fig. 1. The cross-sectional aspect ratio and the $200 \mathrm{~mm}$ gauge length met the requirements set out in flexural testing standards. ${ }^{19}$ Moreover, the gauge length provided a region in which the gas bubble penetration could develop over a flow path that was 13 sections in length.

Melt entered the main specimen cavity via a runner system. The gas injection needle protruded into the centre of the cavity thickness close to the runner system positioned upstream of the melt flow. Along the gauge length, ejector pins were positioned to prevent the component from sticking inside the cavity during demoulding. Mould temperature control was achieved by circulating temperature controlled water through channels cut into the steel mould plate.

\section{Process and product measurement}

The melt and gas injection processing systems were monitored using a computerised data acquisition system based upon 'Labview' software. This system collects signals from sensors mounted on the injection moulding machine, and the gas injection system. The process signals that were measured were: melt injection screw displacement (from which velocity can be inferred), melt-injection system hydraulic pressure, and gas injection pressure. These signals were available from within the machine control system, however additional precision transducers were added to verify the screw displacement, melt pressure and tool temperature. A magneto-restrictive linear displacement transducer was 
mounted on the actuation system for the screw, and was used to measure the melt injection screw displacement; the displacement was differentiated to determine the screw velocity. The melt injection system hydraulic pressure was also measured directly, since there is a direct correlation to the melt injection pressure. Measuring melt pressure at the machine nozzle is more direct, however a rheometric nozzle is required to house a transducer, and these are open to gas penetration. A non-return nozzle was necessary (to stop gas from penetrating into the machine barrel) which prevented direct measurements of melt pressure in the die. Melt and tool temperature were set as a machine control parameter; independent measurements of the tool revealed deviation of typically $2-3^{\circ} \mathrm{C}$ about the set temperature. The effects of these variations on test products were minimised by using a long production run ( $3 \mathrm{~h}$ from start-up) to ensure a steady state before selecting products for measurement.

The process control parameters investigated are listed in Table 1. The gas delay time is the delay between the end of polymer injection, and the beginning of gas injection; this is commonly used as a device to 'freeze' narrow passages to prevent gas penetration along them, for example thin strengthening ribs connected to a thicker gas channel. 'Gas trigger' refers to the position on the injection screw that was used to trigger the (separate) gas injection unit; in essence this creates a time delay before gas triggering, but with some variation at different screw speeds due to the polymer injection screw slowing near the end of its travel.

\section{Product measurement}

An image analysis technique was developed in order to accurately analyse a specimen's cross-section, and measure the residual wall thickness, for correlation with processing parameters. The aim of the system is to give a consistent measurement, avoiding variation associated with human interpretation. The resolution of the image analysis system is nominally $0.02 \mathrm{~mm}$, which is comparable with a measurement from a Vernier calliper. Product to product variation at each setting was reduced by averaging over 10 samples.

A short cross-section of the gauge length was removed using a fine toothed band saw at a position $50 \mathrm{~mm}$ from the beginning of the gauge length, as indicated in Fig. 1. The cross-section was scanned, and the resulting image was analysed to determine the wall thickness in specific locations. Figure 2 shows a typical scanned cross-section showing the gas bubble through the centre of the rectangular section. The image analysis software located the central axes of the rectangular form and determined the residual wall thickness (RWT) along those axes at the four locations indicated.

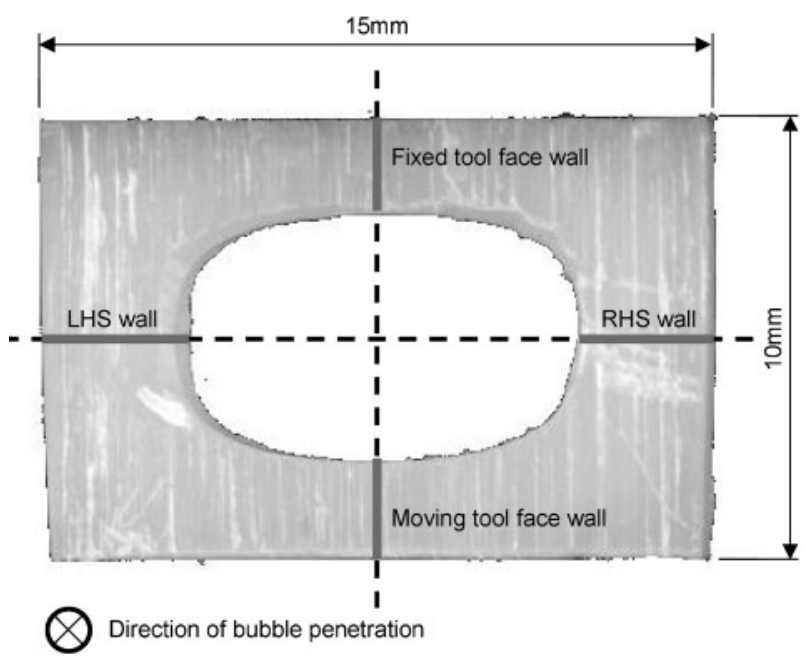

2 Cross-section of manufactured product after cutting, showing measurement positions for residual wall thicknesses

The locations at which the residual wall thicknesses were measured are labelled according to the position inside the mould. The faces in contact with the moving and fixed sides of the mould are referred to as 'moving' and 'fixed' residual wall thicknesses, respectively. The left and right hand walls are referred to as 'LHS' and 'RHS', respectively.

\section{Experimental design}

From consideration of the process, and initial experiments, the residual wall thickness was expected to depend most strongly upon the six processing parameters listed in Table 1. Product variation at any given setting indicated that averaging would be required; a 'design of experiment' approach was indicated both to reduce the number of different measurements, and to identify which processing parameters had a statistically significant effect on wall thickness.

An orthogonal array method was used to select the experiments required to characterise the relationship between the product RWT and the process parameter settings. The framework is an 18-experiment ('Level 18') method where a response against up to eight processing factors can be studied. ${ }^{20}$ This level allows one of these factors to be studied at two 'levels' and seven factors to be studied at three 'levels'; two levels requires measurements at the minimum and maximum of the range of interest giving an implied linear relationship, whereas three levels require measurements covering minimum, maximum, and central values, and a quadratic relationship can be established (full details of the methodology used are given in Refs. 20 and 21). The resulting ' $\left(2^{1} \times 3^{7}\right)$ ' array is detailed in Table 2 . From the standard

Table 1 Process parameters included in experimental study

\begin{tabular}{lll}
\hline Description & Abbreviation for normalised value & Control method \\
\hline Set tool temperature & STT & Closed loop control of coolant \\
Set melt temperature & SMT & Injection moulding machine controller \\
Melt injection velocity & IV & Injection moulding machine controller \\
Gas injection pressure & GP & Gas injection unit controller \\
Gas delay time & GD & Gas injection unit controller \\
Gas controller trigger & GT & Injection moulding machine controller \\
\hline
\end{tabular}


array, the quadratic components of the factors studied at three levels can be determined to quantify the nonlinear component in the response. These normalised level settings are coded $-1,0$ and +1 to define the low, middle and high settings, respectively. For example, Set Tool Temperature can vary from 30 to $60^{\circ} \mathrm{C}$, its normalised equivalent, STT, can take values $-1,0$, and +1 corresponding to $30^{\circ}, 45^{\circ}$, and $60^{\circ} \mathrm{C}$, respectively. This coding system simplifies the multiplication of columns to derive orthogonal interaction columns that are used to investigate interactions between processing parameters on the response (i.e. the RWT). ${ }^{20,21}$ The parameters $e 1$ and $e 2$ are 'empty', notional factors which have no effect on residual wall thickness, and can be used as 'control' parameters to assess the degree of noise in the result; their apparent significance sets a baseline for true physical changes.

Statistical tests were applied to the results to determine which terms were significant, including tests on interaction terms between control variables. The criteria for a term being significant was that it should contribute more than two of the standard deviations of the noise $\left(T_{\text {stat }}>2\right)$, and have a probability of being random of less than $5 \%(P$ value $<0.05)$; see Ref. 20 for details of the procedure. Factors that failed these tests were omitted from the final regression analysis, since their inclusion obscures which factors are important, and tends to provide a fit to noise within the data.

\section{Table 2 L18 $\left(2^{1} \times 3^{7}\right)$ array, showing settings of normalised process control parameters for prescribed 18 experiments}

\begin{tabular}{|c|c|c|c|c|c|c|c|c|}
\hline \multirow[b]{2}{*}{ Run } & \multicolumn{8}{|c|}{ Main factors } \\
\hline & STT & SMT & IV & GP & GD & GT & e1 & $\mathrm{e} 2$ \\
\hline 1 & -1 & -1 & -1 & -1 & -1 & -1 & -1 & -1 \\
\hline 2 & -1 & -1 & 0 & 0 & 0 & 0 & 0 & 0 \\
\hline 3 & -1 & -1 & 1 & 1 & 1 & 1 & 1 & 1 \\
\hline 4 & -1 & 0 & -1 & -1 & 0 & 0 & 1 & 1 \\
\hline 5 & -1 & 0 & 0 & 0 & 1 & 1 & -1 & -1 \\
\hline 6 & -1 & 0 & 1 & 1 & -1 & -1 & 0 & 0 \\
\hline 7 & -1 & 1 & -1 & 0 & -1 & 1 & 0 & 1 \\
\hline 8 & -1 & 1 & 0 & 1 & 0 & -1 & 1 & -1 \\
\hline 9 & -1 & 1 & 1 & -1 & 1 & 0 & -1 & 0 \\
\hline 10 & 1 & -1 & -1 & 1 & 1 & 0 & 0 & -1 \\
\hline 11 & 1 & -1 & 0 & -1 & -1 & 1 & 1 & 0 \\
\hline 12 & 1 & -1 & 1 & 0 & 0 & -1 & -1 & 1 \\
\hline 13 & 1 & 0 & -1 & 0 & 1 & -1 & 1 & 0 \\
\hline 14 & 1 & 0 & 0 & 1 & -1 & 0 & -1 & 1 \\
\hline 15 & 1 & 0 & 1 & -1 & 0 & 1 & 0 & -1 \\
\hline 16 & 1 & 1 & -1 & 1 & 0 & 1 & -1 & 0 \\
\hline 17 & 1 & 1 & 0 & -1 & 1 & -1 & 0 & 1 \\
\hline 18 & 1 & 1 & 1 & 0 & -1 & 0 & 1 & -1 \\
\hline
\end{tabular}

Factor settings

\begin{tabular}{llllllll}
\hline $\begin{array}{l}\text { STT, } \\
{ }^{\circ} \mathbf{C}\end{array}$ & $\begin{array}{l}\text { SMT, } \\
{ }^{\circ} \mathbf{C}\end{array}$ & $\begin{array}{l}\text { IV, } \\
\mathbf{m m ~ s}^{-1}\end{array}$ & $\begin{array}{l}\text { GP, } \\
\text { bar }\end{array}$ & $\begin{array}{l}\text { GD, } \\
\text { s }\end{array}$ & $\begin{array}{l}\text { GT, } \\
\text { mm }\end{array}$ & e1 & e2 \\
30 & 210 & 20 & 30 & 0 & $0 \cdot 2$ & $\ldots$ & $\ldots$ \\
$\ldots$ & 230 & 50 & 55 & 5 & $0 \cdot 6$ & $\ldots$ & $\ldots$ \\
60 & 250 & 80 & 80 & 10 & $1 \cdot 0$ & $\ldots$ & $\ldots$ \\
\hline
\end{tabular}

The physical values of control settings corresponding to the normalised values of control settings are shown. Full names can be read from Table 1, e1 and e2 are empty factor columns which help quantify noise in the experiments.

\section{Experimental results}

Experiments were carried out over the range of process parameters, as prescribed by the Level 18 method. After tests to determine which terms were statistically significant, a polynomial was obtained for the residual wall thicknesses for the 'LHS' or 'RHS' walls, and the 'Fixed' or 'Moving' walls.

A total of 180 experiments were undertaken in all, 10 at each of the 18 experimental settings to reduce noise and natural variation. The equation for the averaged wall thickness for the LHS-RHS walls, $\mathrm{RWT}_{\mathrm{lhs} / \mathrm{rhs}}$, was found to be

$$
\begin{aligned}
\mathrm{RWT}_{\mathrm{lhs} / \mathrm{rhs}} & =2 \cdot 9261+0 \cdot 2372(\mathrm{GD})-0 \cdot 1062\left(\mathrm{GD}^{2}\right) \\
& -0 \cdot 1009(\mathrm{IV})-0 \cdot 0926(\mathrm{SMT})
\end{aligned}
$$

and the equation for the averaged wall thickness for the Fixed-Moving walls, $\mathrm{RWT}_{\text {fix/mov }}$, was found to be

$$
\begin{aligned}
& \mathrm{RWT}_{\mathrm{fix} / \mathrm{mov}}=1 \cdot 9254+0 \cdot 2672(\mathrm{GD})-0 \cdot 0600\left(\mathrm{GD}^{2}\right) \\
&-0 \cdot 0453(\mathrm{GP})-0 \cdot 0435(\mathrm{IV})-0 \cdot 0377(\mathrm{STT})
\end{aligned}
$$

In both of these equations, it is the normalised value which should be input (for example, $-1 \leqslant \mathrm{GD} \leqslant 1$ ), the output value is the wall thickness $(\mathrm{mm})$. The gas trigger delay (GT) variable was identified by the statistical procedure as being statistically insignificant, and does not appear in either equation. This is reasonable, as it is equivalent to a maximum possible adjustment of only $0 \cdot 2 \mathrm{~s}$ in the gas delay time. Comparison between the two models, and the original 18 experimental settings gave an average error of $0.03 \mathrm{~mm}$, which represents an average error in percentage residual wall thickness of $0.6 \%$. The peak residual error in fitting any experiment was $0.1 \mathrm{~mm}$, which is seen to be small compared with the range of residual wall thicknesses in figures that follow.

For both pairs of walls, gas delay time (GD) was the most significant parameter, and clearly non-linear (as might be expected from the 'square root' relationship relating depth penetrated by heat to time available, as shown later in this work). Injection velocity (IV) features in both, and this will also have an effect on effective cooling time, but during the melt injection phase. Gas pressure (GP) will affect both cooling time and shear distribution; this gave statistical significance on the Fixed-Moving walls, but was not pronounced enough to register as significant on the LHS-RHS walls.

\section{Heat transfer considerations}

Heat transfer is a key factor for understanding many of the experimental results, and for simulation of the GAIM process. As an injection-moulding mould normally has a much higher conductivity than polymer, the mould might reasonably be assumed to be at a single temperature, close to the coolant temperature. Figure 3 shows the situation at the polymer melt/mould wall interface. It is common is such situations to use a heat transfer coefficient, however, such values are notoriously difficult to measure with any accuracy and, as will be shown, may not be appropriate for transient thermal problems such as when a hot polymer melt touches a cool metal wall. The accuracy of a method that sets the temperature boundary to be the fixed coolant temperature was investigated, when polymer first contacts the 


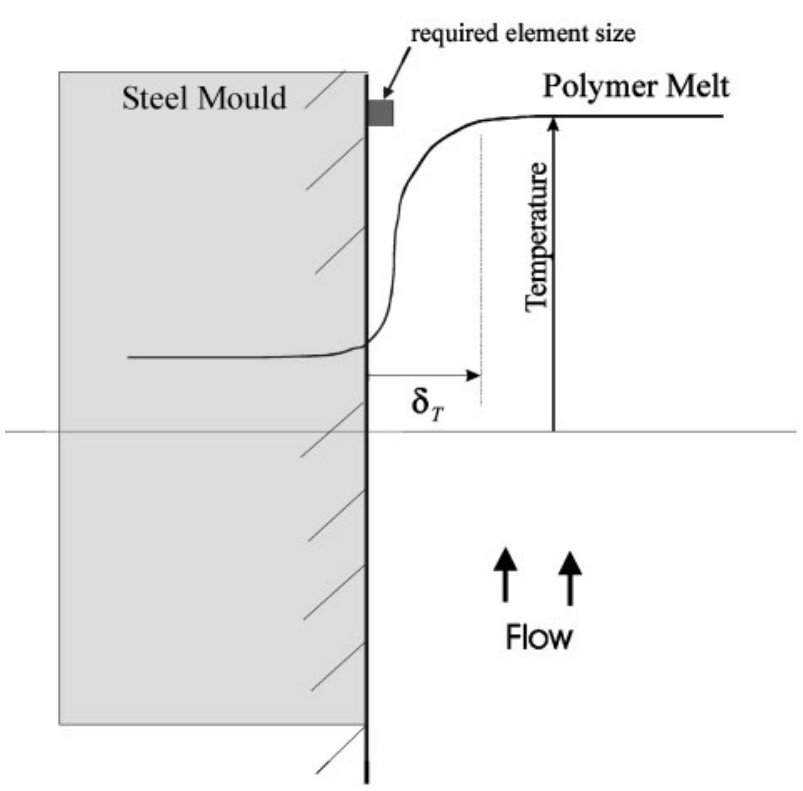

3 Variation in temperature at mould/polymer interface

mould wall. For the method to be accurate the finite element system must be able to accurately compute the heat exchange at the wall, and the difference between the mould-wall temperature and the controlling coolant temperature, must be small. For accurate heat flux calculation, the finite element solution must to be able to determine the temperature gradient normal to the wall; this requires that the element size at the wall is significantly less than any thermal boundary layer thickness. Thus, three situations need examining: the penetration depth upon initial polymer-mould contact, the thermal boundary layer due to any flow, and the change in heat flux when variation in die temperature is considered.

\section{Transient heat penetration}

The situation whereby a fixed temperature boundary condition is applied when polymer first comes into contact with the mould wall is very close to a 'standard' problem, which has a known analytical solution. If a 'semi-infinite' solid at an initial temperature $T_{\mathrm{i}}$ has a surface set to a temperature $T_{\mathrm{f}}$ at a time $t=0$, then the temperature at a depth $x$, at a time $t$, is given by

$$
\frac{T(x, t)-T_{\mathrm{i}}}{T_{\mathrm{f}}-T_{\mathrm{i}}}=\operatorname{erfc}\left(\frac{x}{2 \sqrt{\alpha t}}\right)
$$

where $\operatorname{erfc}()$ is the Complimentary Error Function and $\alpha$ (the thermal diffusivity) is given by $\alpha=\sigma / \rho C_{\mathrm{p}} \cdot{ }^{22}$ Taking $50 \%$ of the potential temperature change as indicative of 'heat penetration' (and noting that $\operatorname{erfc}(0 \cdot 5) \approx 0 \cdot 5$ ) leads to: $x_{50 \%} \approx \sqrt{\alpha t}$, where $x_{50 \%}$ is the depth to which the $50 \%$ change in temperature has penetrated after a time $t$. Using the thermal properties for HDPE 5050EA gives

$$
x_{50 \%} \approx 5 \times 10^{-4} t^{1 / 2}
$$

As will be described, the mesh used in simulation has an element size of approximately $0.2 \mathrm{~mm}$ near the wall, implying that the thermal gradient will be well resolved by the mesh when the heat penetrates to $0.2 \mathrm{~mm}$. From equation (4), the finite element solution will be able to accurately calculate wall heat flux after an initial $\sim 0.2 \mathrm{~s}$;
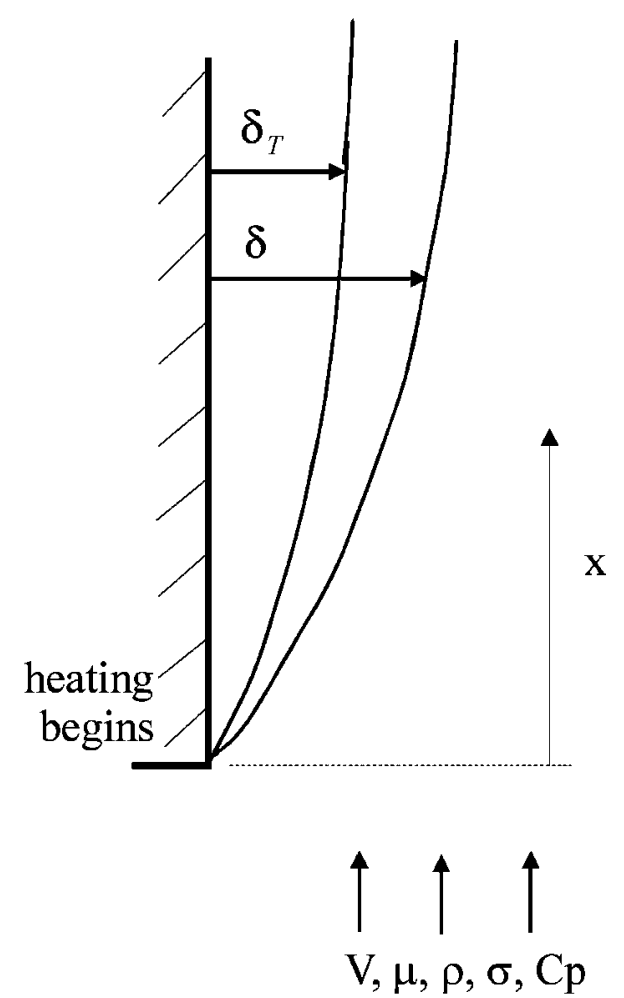

4 Development of momentum and thermal boundary layer along wall

before this time, the heat flux calculation may not be accurate. This prediction of time before accurate computation is confirmed in a following section. It is also shown that the resulting error is small for the mesh used.

\section{Flow related thermal boundary layer}

In the experiments performed, the flow related thermal boundary layer is thinnest during gas injection when the polymer is moving fastest. Assuming that steady state boundary layer conditions are appropriate for the heat transfer processes, then the situation represented in Fig. 4 is present. The thermal boundary layer thickness $\delta_{\mathrm{T}}$ is calculated from

$$
\frac{\delta_{\mathrm{T}}}{\delta}=\frac{1}{1 \cdot 026} \operatorname{Pr}^{-\frac{1}{3}}
$$

where $\operatorname{Pr}$ is the Prandtl number, $v / \alpha$, and $\delta$ is the velocity boundary layer thickness, given by $\delta=5 x /\left(\operatorname{Re}_{\mathrm{x}}\right)^{\frac{1}{2}}{ }^{23}$ In these equations, $v$ is the kinematic viscosity, $\alpha$ is equal to $\sigma / \rho C_{\mathrm{p}}$ where $\sigma$ is conductivity, $\rho$ is density, and $C_{\mathrm{p}}$ is specific heat capacity; $\operatorname{Re}_{\mathrm{x}}$ is the local Reynolds number given by $\mathrm{Re}_{\mathrm{x}}=\rho V x / \mu$ where $V$ is the free stream velocity, and $x$ is the distance along the wall from the point where heat transfer began. ${ }^{23}$

Combination of these terms leads to

$$
\delta_{\mathrm{T}} \approx 5 \mu^{\frac{1}{6}}\left(\frac{\sigma}{C_{\mathrm{p}}}\right)^{\frac{1}{3}}\left(\frac{x}{\rho V}\right)^{\frac{1}{2}}
$$

For the polymer used in the experiment, values (from manufacturer's data) of $\rho=950 \mathrm{~kg} \mathrm{~m}^{-3}, \quad C_{\mathrm{p}}=$ $2300 \mathrm{~J} \mathrm{~kg}^{-1} \mathrm{~K}^{-1}, \mu=1.2 \times 10^{3} \mathrm{~Pa} \mathrm{~s}$ (typically), and $\sigma=$ $0.48 \mathrm{~W} \mathrm{~m}^{-1} \mathrm{~K}^{-1}$ are obtained. In the GAIM experiment, the melt flow velocity was always less than 


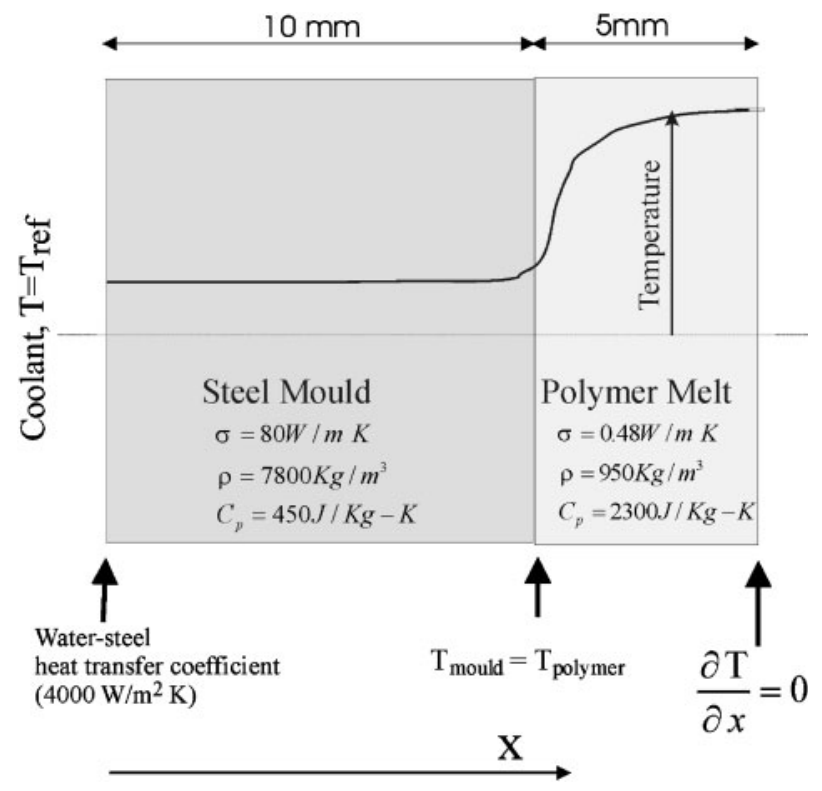

5 Geometry and boundary conditions for finite-difference computations

$1 \mathrm{~m} \mathrm{~s}^{-1}$. Taking this value as the worst case, and setting $V$ to be $1 \mathrm{~m} \mathrm{~s}^{-1}$ in equation (6) gives $\delta_{\mathrm{T}} \approx 3 \mathrm{~mm}$, when $x=1 \mathrm{~cm}$, and $\delta_{\mathrm{T}} \approx 10 \mathrm{~mm}$ when $x=10 \mathrm{~cm}$; thus a boundary mesh size of approximately $0.2 \mathrm{~mm}$ should suffice to accurately capture the heat loss over the great majority of the contact area in the mould.

\section{Mould wall temperature, and directly computed heat flux}

To assess errors in the assumption that the mould wall was at the same temperature as the cooling water, a high resolution finite difference computation was performed (on a $0.01 \mathrm{~mm}$ grid). From knowledge of the construction of the die, $1 \mathrm{~cm}$ was taken as a characteristic distance from mould/polymer interface to coolant, and $5 \mathrm{~mm}$ was taken as a characteristic half-depth of polymer. The situation is shown in Fig. 5. The initial temperature of polymer was set to be $230^{\circ} \mathrm{C}$, and the steel was set to an initial temperature of $45^{\circ} \mathrm{C}$, as used in most simulations. The boundary conditions and thermal properties of polymer and steel are shown in Fig. 5, and a heat transfer coefficient of $4 \mathrm{~kW} \mathrm{~m} \mathrm{~K}^{-2}$ was used for the coolant/mould interface, as used in Refs. 17 and 18. Implicit time-stepping was used to progress the system in time, and the heat transfer coefficient (referenced to the $45^{\circ} \mathrm{C}$ reservoir temperature), and the heat fluxes were calculated. These calculated values are referred to as 'true' values. The computation was repeated with the mould/polymer interface set to the coolant temperature, as is being considered for the GAIM simulations. The heat fluxes were again calculated (the heat transfer coefficient here is infinity, by definition). These heat fluxes are referred to as fixed-temperature heat fluxes. The 'true' heat transfer coefficients obtained are shown in Fig. 6. These are initially of order $10^{5}$ and remain over $10^{4}$ until after $10 \mathrm{~s}$. Figure 7 shows the 'true' and 'fixed-temperature' heat fluxes. These are almost inseparable to graphical scale (from detailed examination, the fixed temperature fluxes are typically 1-2\% higher). It is interesting to note that, despite the difference in heat transfer coefficient, the heat fluxes are very nearly identical.

The two computations were repeated, but using a much coarser grid to match the GAIM simulation resolution $(0 \cdot 2 \mathrm{~mm}$ at the wall). The lower two curves in Fig. 7 show the situation: very similar results are

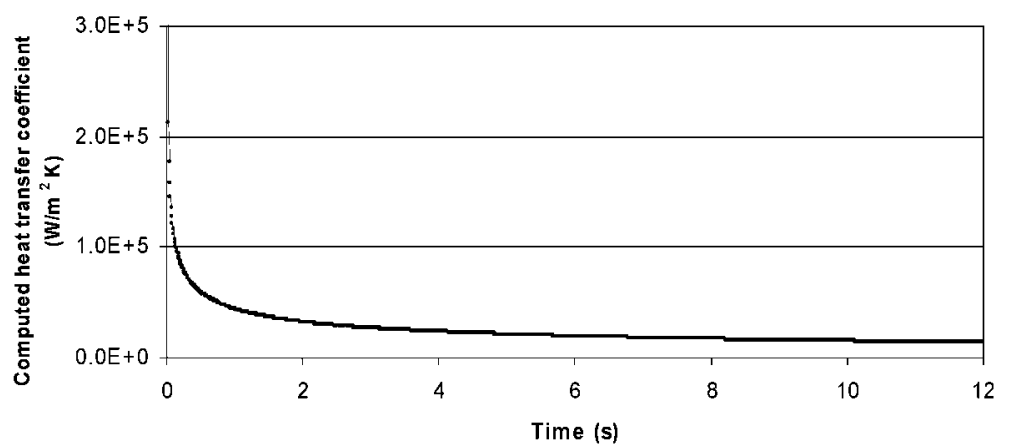

6 Computed transient heat transfer coefficients, referenced to coolant temperature, upon polymer contacting mould wall

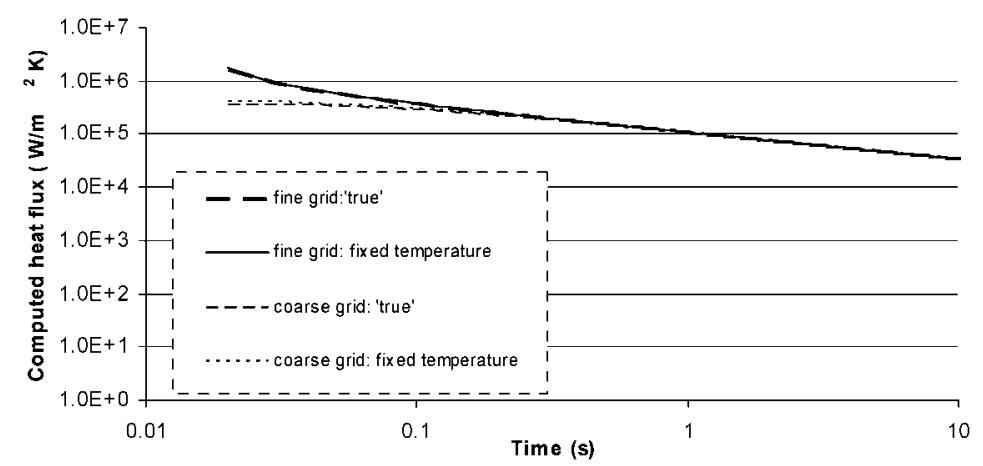

7 Comparison between wall heat fluxes computed with 'true', and 'fixed-temperature' boundary conditions; computations are shown for both a fine grid $(0.01 \mathrm{~mm})$, and a coarse grid $(0.2 \mathrm{~mm})$ 
obtained for the 'true' boundary conditions and the 'fixed-temperature' boundary conditions. Both of the coarse grid curves are below the fine grid curves until shortly after $0.1 \mathrm{~s}$; this time interval confirms the analytical prediction from equation (4) $(\sim 0 \cdot 2 \mathrm{~s})$. The errors inherent in using a finite mesh are small, but for the 1-D case are clearly much greater than the errors due to using the 'fixed-temperature' boundary conditions.

Using the 'true' boundary conditions, the temperature rise (above reservoir temperature) at the polymer/mould interface was found to be only $3 \cdot 5^{\circ} \mathrm{C}$ after $0 \cdot 1 \mathrm{~s}$ of contact, and $2 \cdot 5^{\circ} \mathrm{C}$ after $1 \mathrm{~s}$ of contact. The closely matching heat fluxes, and the small changes in tool temperature (in conjunction with the insensitivity of residual wall thicknesses to changes in tool temperature that is demonstrated later) indicate that the 'fixedtemperature' boundary condition is an accurate approximation to the true thermal conditions, particularly for residual wall thickness computation.

\section{Numerical modelling}

To simulate the GAIM process in 3-D, WE employ the 'pseudo-concentration' method. ${ }^{8-10}$ This involves a time-stepping procedure whereby the polymer 'concentration' is convected with the flow in small time increments. The model uses a scheme in which the polymer has a concentration value, $c$, of unity, and the injection gas (often called a 'fictitious fluid') has a concentration value of zero. The viscosity, $\mu$, at a point is a function of the concentration, i.e. $\mu=f(c)$. In these simulations, linear interpolation is used

$$
\mu=c \mu_{\text {polymer }}+(1-c) \mu_{\text {gas }}
$$

where $\mu_{\text {polymer }}$ is the viscosity of polymer, and $\mu_{\text {gas }}$ represents the viscosity of the gas. As a result of the large difference between physical values of $\mu_{\text {polymer }}$ and $\mu_{\text {gas }}$, a significant loss of accuracy can occur in numerical simulations due to numerical rounding errors. Because of this, it is necessary to moderate the ratio of the two viscosities. ${ }^{9}$ We set $\mu_{\text {gas }}$ according to

$$
\frac{\mu_{\text {gas }}}{\mu_{\text {polymer }}}=\varepsilon
$$

where $\varepsilon$ is a small number of order $10^{-3}$ or lower. Results for residual wall thickness have been shown to be virtually independent of $\varepsilon$, provided that $\varepsilon$ is sufficiently small. ${ }^{9}$ Concentration, $c$, is transported according to the convective flow equation for immiscible substance, thus

$$
\frac{D c}{D t}=\frac{\partial c}{\partial t}+\boldsymbol{u} \cdot \nabla c=0
$$

where $\boldsymbol{u}$ is the flow velocity.

This purely convective concentration transport equation requires some kind of stabilisation; a form of upwinding is used that provides diffusion in the direction of flow. ${ }^{24}$ Upwinding methods are discussed in detail in Ref. 25.

In the pseudo-concentration method, it is normally assumed that the flow at a point has a Newtonian form and (neglecting body-forces, and convection terms) obeys

$$
\nabla \cdot\left(\mu\left(\mathbf{L}+\mathbf{L}^{\mathbf{T}}\right)-\mathbf{I} p\right)=\rho \frac{\partial \boldsymbol{u}}{\partial t}
$$

where $\mu$ is viscosity, $\rho$ is density, $p$ is pressure, $\mathbf{I}$ is the identity matrix tensor, and $\mathbf{L}$ is the velocity gradient tensor, defined by $L_{i j}=\partial u_{i} / \partial x_{j}$. Additionally, incompressibility is enforced according to $\nabla \cdot \boldsymbol{u}=0$.

The temperature field evolves according to the thermal transport equation which (neglecting viscous dissipation) is given by

$$
\rho C_{\mathrm{p}}\left(\frac{\partial T}{\partial t}+\boldsymbol{u} \cdot \nabla T\right)=\nabla \cdot(\sigma \nabla T)
$$

We use the Galerkin finite element formulation to solve the momentum, incompressibility, concentration, and thermal transport equations. Velocity components, temperature, and concentration are approximated using $\widetilde{v}=\sum v_{\mathrm{j}} \psi_{\mathrm{j}}$, where $\tilde{v}$ is the interpolated value, $v_{j}$ are the nodal values of the variable in the element being considered, and $\psi_{\mathrm{j}}$ are the nodal interpolation functions. $\psi_{\mathrm{j}}$ are eight-node 'brick' interpolation functions in the 3-D simulations. A penalty pressure method is used in most simulations of the method. Pressure is treated as an independent variable using interpolation functions $\phi_{\mathrm{j}}$ of one order lower than for velocity components: $\widetilde{p}=\sum \phi_{\mathrm{j}} p_{\mathrm{j}}$.

For realistic representation of polymer injection, a non-slip boundary condition may be used for walls that are in contact with polymer; a zero normal flow boundary condition, $\boldsymbol{n} \cdot \boldsymbol{u}=0$ (where $\boldsymbol{n}$ is the vector normal to the wall), has often been used on gas/wall interfaces. ${ }^{9,26}$ We adopt the method in Ref. 17 and leave the flow unconstrained at gas-wall boundaries. This method has been shown to give a realistic representation of fountain flow, ${ }^{17}$ and avoids problems of corners into which polymer cannot flow. At each time-step, 'clipping' is applied to the nodal values obtained for concentration, $c$ : those values that are above 0.5 are set to unity, and values that are below 0.5 are set to zero. This is applied to all nodes, except to nodes that are in an element through which the $c=0 \cdot 5$ contour passes (if this exception is not applied, then the small movement of the polymer front during a single time step is largely cancelled by the clipping). Elements through which the ' $c=0 \cdot 5$ ' contour passes can be easily identified since they will have at least one node with a concentration value greater than $0 \cdot 5$, and at least one node for which the concentration value is below $0 \cdot 5$. The interface between polymer and gas is taken to be the contour of $c=0 \cdot 5$.

\section{Thermal boundary conditions}

The thermal boundary conditions are critical to the temperature evolution, and subsequent residual wall thickness. Following the study in the section 'Heat transfer considerations' above, the temperature at the polymer/mould interface is set to be equal to the set tool temperature. That is, at the wall, $T_{\text {polymer }}=T_{\text {mould }}$. The accuracy of the assumptions made were assessed for a 1-D characteristic system in the section 'Heat transfer considerations' above, and found to give a good approximation to the 'true' heat fluxes.

A feature of this method is that it leaves the solution procedure to calculate heat transfer according to the flow conditions and heat transport equations. It also inherently assumes good thermal contact between wall and polymer, and gives the greatest rate of cooling that 


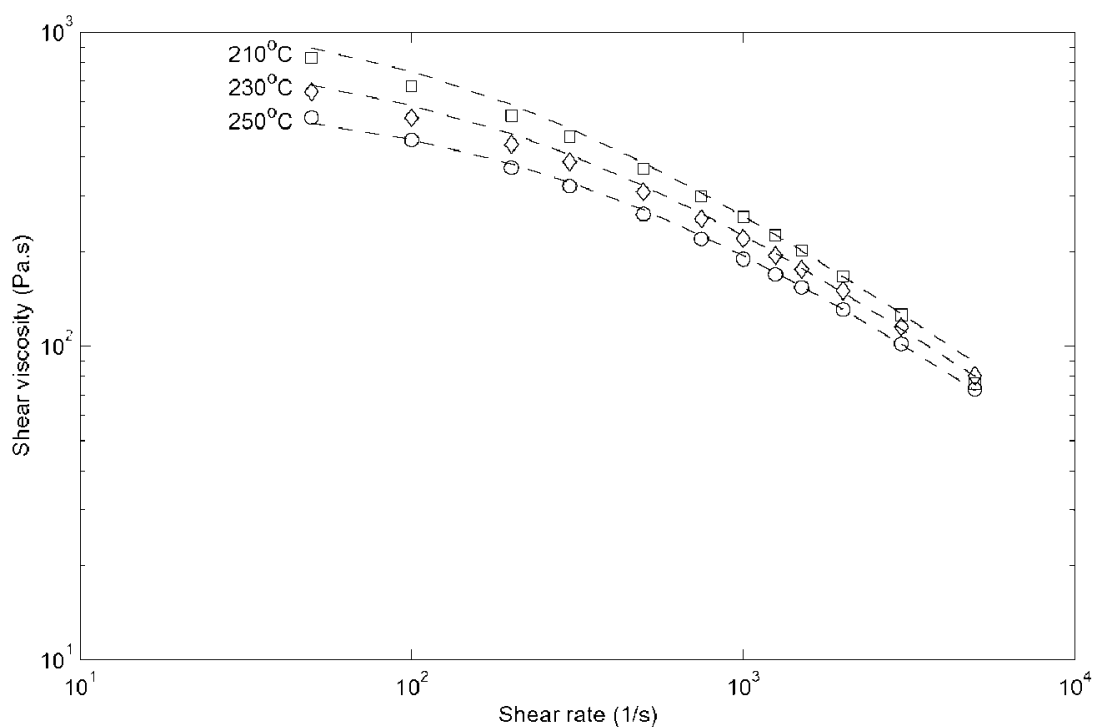

8 Comparison between experimental rheometric data for BP HDPE 5050EA, and Cross-WLF model used in simulations

can physically happen; this 'upper bound' knowledge is useful, later, in analysing results.

\section{Temperature and shear dependence}

We employ a form of the Cross-WLF model to incorporate viscosity temperature dependence. This takes the form

$$
\mu_{\text {polymer }}=\frac{\mu_{\mathrm{s}} a(T)}{1+(\lambda a(T) \dot{\gamma})^{1-\mathrm{n}}}
$$

where

$$
\log (a(T))=\frac{-c_{1}\left(T-T_{\mathrm{s}}\right)}{c_{2}+T-T_{\mathrm{s}}}
$$

In this model, $a(T)$ is a WLF shift factor, $\dot{\gamma}$ is shear rate, $n$ is the power law exponent, $\mu_{\mathrm{s}}$ is the polymer viscosity at reference temperature $T_{\mathrm{s}}$, and $\lambda, c_{1}$, and $c_{2}$ are empirically determined constants. $\mu_{\text {polymer }}$ is then the polymer viscosity at temperature $T$ and shear rate $\dot{\gamma}^{18,27}$

The parameters for equations (12) and (13) were obtained by fitting experimental data for high-density polyethylene (HDPE) (BP Rigidex 5050EA) obtained from capillary rheometry. A natural logarithm base was used for equation (13); the resulting parameters, used in all simulations, are $\mu_{\mathrm{s}}=1 \cdot 228 \times 10^{3} \mathrm{~Pa} \mathrm{~s}, T_{\mathrm{s}}=210^{\circ} \mathrm{C}$, $\lambda=5.58 \times 10^{-3} \mathrm{~s}, \quad n=0.23335, \quad c_{1}=17 \cdot 44, \quad$ and $c_{2}=$ $1000^{\circ} \mathrm{C}$. Figure 8 shows the fit of the model to the experimental data.

\section{Needle injected gas source}

The injection of polymer has previously been simulated by setting the nodes on the inlet boundary to have a concentration value of 1 ; this can be later changed to 0 to simulate gas injection. However, the current process (and most industrial GAIM processes) injects gas from a small needle which is surrounded by polymer melt in the mould. Gas from this needle is thus used to propel, and 'core-out' the polymer. Figure 9 indicates the two situations. To simulate the industrial process more closely, a source term $S$ is introduced into the mass conservation equation in a single localised volume

$$
\nabla \cdot \boldsymbol{u}=\frac{S}{\Delta}
$$

where $\Delta$ is the volume of the space in which the gas source is positioned. In this form, the numerical value of $S$ then sets the volume flowrate being injected into the element.

In initial experiments, one (or several) whole finite element was used as an injection source. This was found to have a significant problem - in typical elements, the volume flow clearly 'preferred' to come out of the element in some directions more than others. This caused non-physical results in simulations; the problem did not reduce sufficiently when the gas source was extended to cover several elements.

Using a spherical volume of Gauss points as an injection source proved much more satisfactory; here an injection point, $\left(x_{\mathrm{c}}, y_{\mathrm{c}}, z_{\mathrm{c}}\right)$, and a source radius $r_{\mathrm{c}}$ are defined. The injection volume of the element $\Delta$ is computed by a volume integration over all elements, of a Gauss-point variable $h$, i.e.

$$
\Delta=\int_{\text {all elements }} h_{\mathrm{k}} \mathrm{d} V
$$

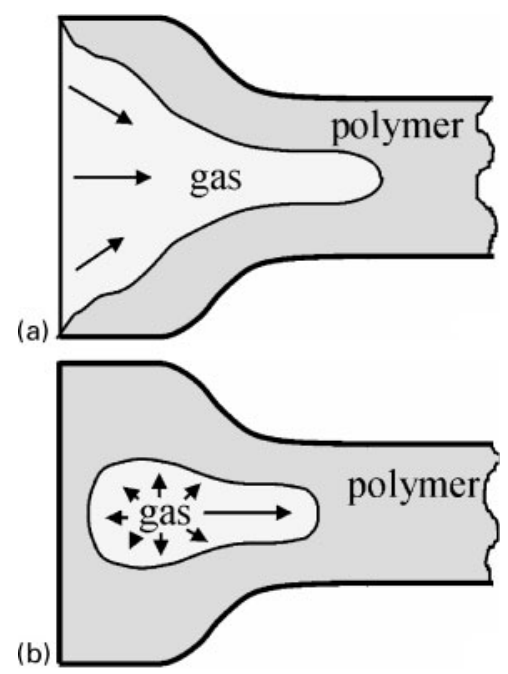

a Injection from boundary; $b$ Injection from internal point

9 Gas injection methods 


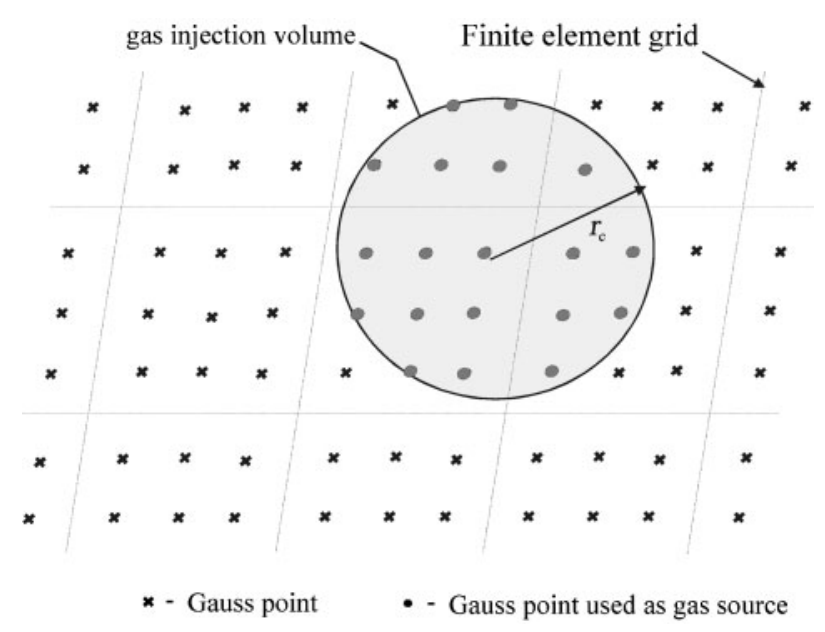

10 Schematic diagram of spherical gas source, and Gauss points at which the source is implemented

where $h_{k}=1$ if the Gauss point $\mathrm{k}$ is within the injection radius and $h_{\mathrm{k}}=0$ if Gauss point $\mathrm{k}$ is outside the injection radius. For a Gauss point $\mathrm{k}$ at position $\left(x_{\mathrm{k}}, y_{\mathrm{k}}, z_{\mathrm{k}}\right)$, the condition for being inside the injection sphere is thus

$$
\left(x_{\mathrm{k}}-x_{\mathrm{c}}\right)^{2}+\left(y_{\mathrm{k}}-y_{\mathrm{c}}\right)^{2}+\left(z_{\mathrm{k}}-z_{\mathrm{c}}\right)^{2} \leqslant r_{\mathrm{c}}^{2}
$$

The method needs to be applied to a volume with a radius at least that of local element dimensions, to give a smooth sphere of Gauss points at which gas injection is applied. The localised source is represented in Fig. 10. Unlike the previous method, this method gives a volume flow without any noted preference for direction. Calculating the volume represented by the 'source' Gauss points, as shown, is preferable to relying on ' $4 \pi r^{3} / 3$ ', since quantitisation effects from the finite number of Gauss points can give a significant difference for sources of small radius.

\section{Simulation overview}

A 3-D mesh of $2 / 3$ of the length of the tensile testpiece was created using eight-node 'brick' elements. This length was sufficient for the polymer-gas profile to develop. Symmetry in two planes allowed that only $1 / 4$ of this geometry need be meshed, allowing an increase in the density of the mesh within the available computer memory. The surface of the mesh is shown in Fig. 11. The simulation began with gas in the mould, hot polymer melt was then injected from one end to approximate the actual injection process; this injection took place over $1.4 \mathrm{~s}$, with a rate that would just fill the whole gauge length in this time (in accordance with measured injection time and 'hesitation marks' on the actual products). At positions with polymer in contact with the walls, the set tool temperature was applied as the thermal boundary condition; at points where gas was in contact with the walls, an adiabatic condition was applied. This replicates the (assumed) negligible thermal interaction between the advancing polymer front, and the gas in the unfilled mould. The fluid was stationary during the gas delay time, and the polymer cooled according to the conduction component of the thermal transport equation, and the applied thermal boundary conditions.

The gas viscosity was set to be $10^{-3}$ times the initial polymer viscosity, i.e. the polymer viscosity at its original injection temperature, and zero shear. During cooling, the viscosity of the polymer increases according to equation (13). The polymer viscosity was limited to no more than 100 times its initial viscosity to keep the ratio of polymer to gas viscosities to be within $10^{5}$, avoiding problems associated with excessive numerical range. After the gas delay time, the needle gas source was 'switched on' at a volume flowrate of (normally) $1.5 \times 10^{-5} \mathrm{~m}^{3} \mathrm{~s}^{-1}$. A concentration value of zero (for gas) was enforced at all Gauss points within the gas source 'sphere'. The momentum, temperature and concentration values were then time-stepped (using a time step of order (1/10) $\Delta x / V$ where $\Delta x$ is the smallest element dimension, and $V$ is the characteristic velocity in the main gas flow). At each time step, the polymer viscosity at a point was calculated according to equations (12) and (13). The effective viscosity was then modified according to the concentration value at that point (equation (7)). These viscosity values were used

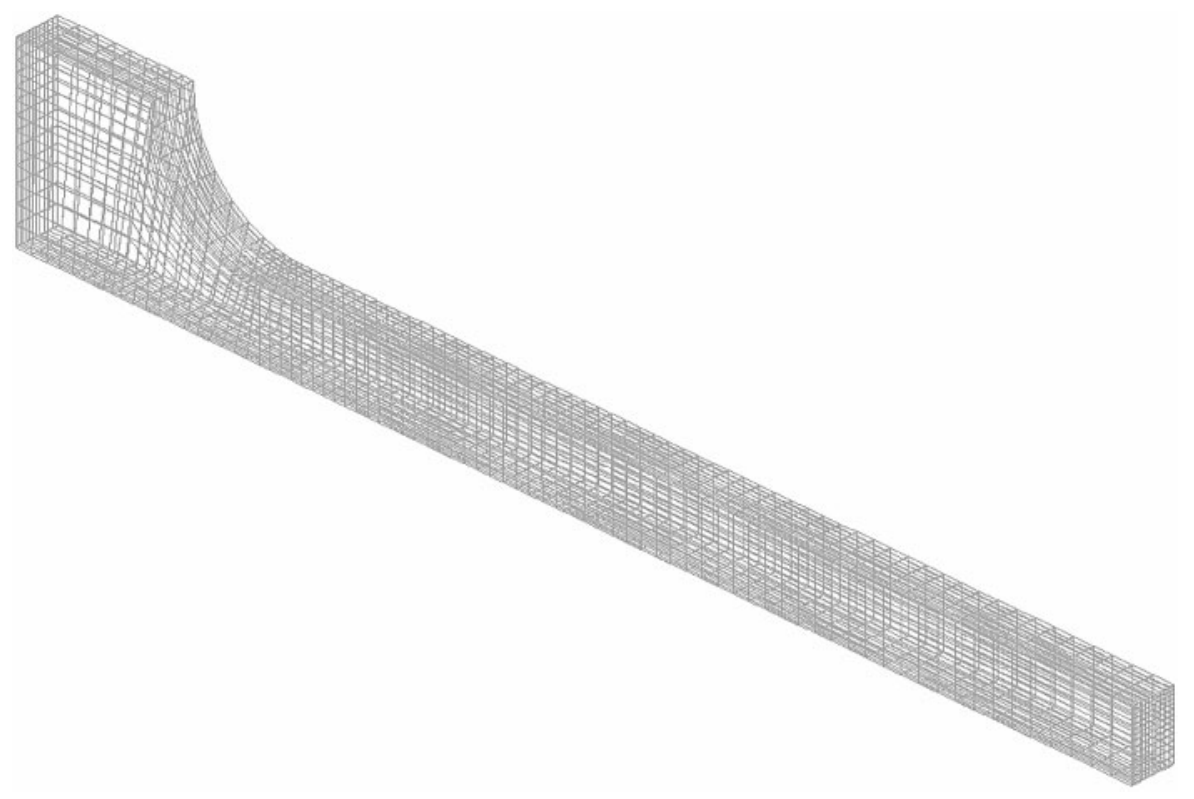

11 Surface of mesh used for simulations 


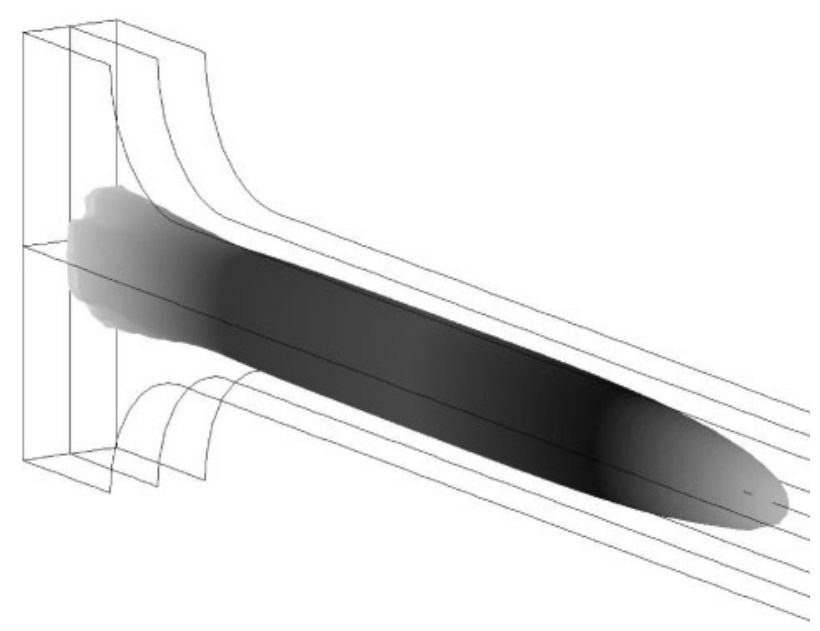

12 Region around gas source and progression of gas bubble through mould, shown by surface of concentration, $c=0.5$; surface shading comes from altering hue according to distance along mould

for the next time step. Figure 12 shows the progress of the gas bubble, represented by the surface of concentration value $c=0.5$; the region around the source is seen to be well behaved, with little roughness, and with no particular direction being preferred. The simulation proceeded until after the gas had reached the end of the mesh, and a fully developed concentration profile had developed. The residual wall thicknesses were measured on the cross-section corresponding to where the experimental measurements were made.

\section{Simulation results and comparisons}

Simulations were made varying gas delay time between 0 and $10 \mathrm{~s}$ (corresponding to $-1 \leqslant G D \leqslant 1$ ), while setting all other parameters to their central value, i.e. $\mathrm{IV}=\mathrm{GP}=\mathrm{SMT}=\mathrm{STT}=0$; the physical values corresponding to these, for use in the simulation can be read from Table 2; the Cross-WLF model was used because shear thinning is to be expected in the high-density polyethylene used in the experiment. The comparison between the value given by the experimentally derived model for the 'fixed' and 'moving' walls, and the LHS and RHS walls (expressed as a percentage of the halfdepth), and the simulation results, is given in Fig. 13.

Comparison between the experimental curves in Fig. 13 shows that the LHS-RHS walls give a greater fractional residual wall thickness than the Fixed-Moving walls at low delay times; at longer delay, the situation reverses. This relative increase is likely to be caused by the penetration of cooling, which is greater (fractionally) for the Fixed-Moving walls. The simulation for the fixed and moving walls agrees well with experiment at delay times up to approximately $2 \mathrm{~s}(<1.5 \%)$, but increases less quickly, compared with experiment, beyond this point. Comparison between experimental and simulated values for the LHS and RHS walls shows a greater difference, with an fairly constant underprediction of $7 \%$; the gradients, however, are seen to match well. When the wall thickness is plotted without normalisation, as shown in Fig. 14, a physically thicker wall is predicted for the LHS/RHS walls. This shows that the depth of the channel is a significant factor in the simulation results, in addition to the penetration depth

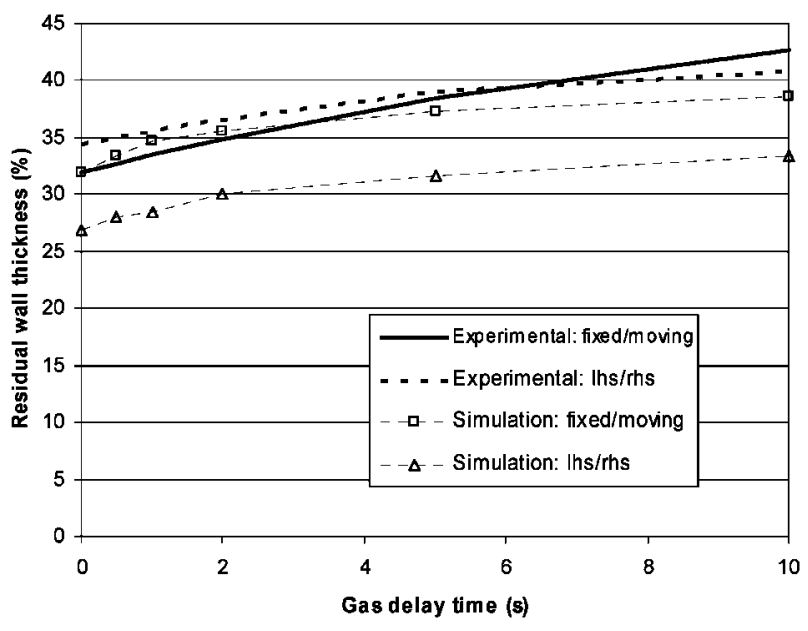

13 Comparison between residual wall thickness from experiment and simulations, as a function of gas delay time

of cooling from the mould walls. Out of interest, the curve for $50 \%$ heat penetration was plotted $\left(x_{50 \%}\right.$, in equation (4)). The gradients were seen to be broadly comparable, and it should be remembered that cooling began in the polymer before the start of gas delay time ( $\sim \mathrm{s})$.

The consistent underprediction of residual wall thickness on the LHS and RHS walls is interesting. If it were considered that the penetration of cooling from the die walls was the dominant factor in causing increased thickness, then insufficient heat transfer at the walls would be considered the most likely cause of this effect. However, due to the use of fixed-temperature boundary conditions, the equivalent heat transfer coefficient would be infinite. This suggests that insufficient mould-polymer heat transfer is unlikely to be the cause. Surface tension is very unlikely to be significant, since the capillary number is $\sim 10^{3}{ }^{6}$ A strong remaining possibility is viscoelasticity in the polymer: stored viscoelastic stresses will relax during the gas delay time (giving gas delay time an effect on RWT that is not predicted by cooling a Cross-WLF fluid), and it seems likely that elastic tension around the gas bubble would tend to increase the LHS and RHS wall thicknesses preferentially. The strong growth of RWT due to elasticity in the flow has already been demonstrated in Ref. 12.

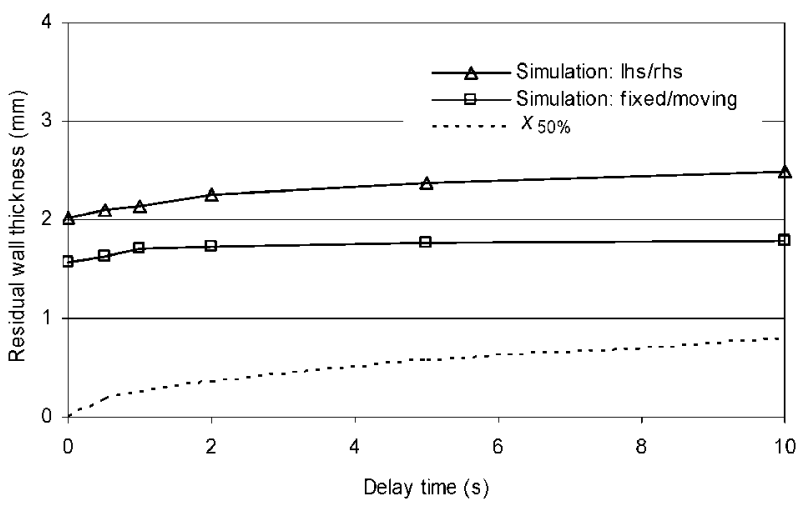

14 Simulation of residual wall thickness, shown in millimetres; analytical solution for depth of $50 \%$ heat penetration (equation (4)) is also shown 

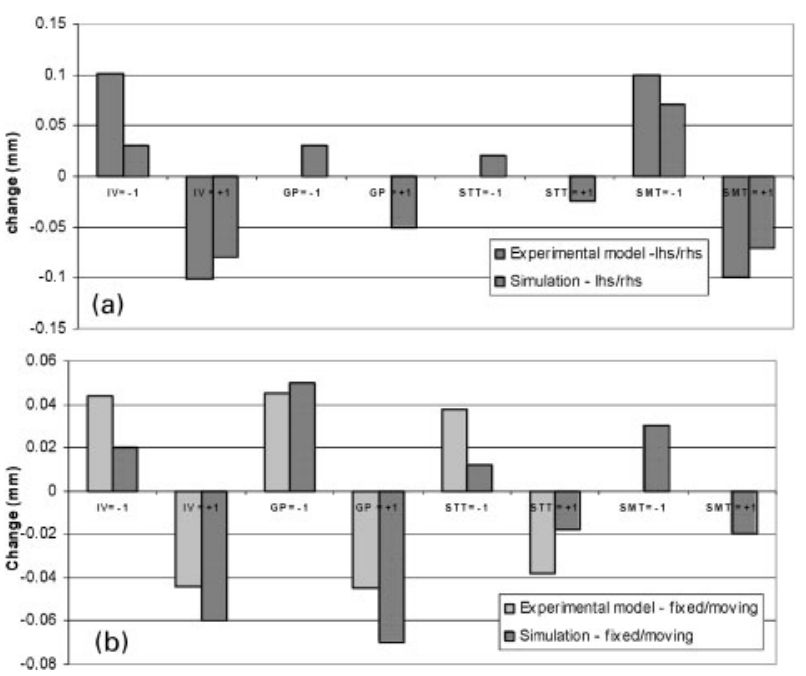

$a$ LHS/RHS walls; $b$ Fixed/moving walls

15 Comparison between changes given by experimentally derived RWT models, and changes predicted by simulation

\section{Simulation prediction of process control changes}

The ability of the simulation to predict the effects of changes in process parameters was studied. The previous results varied gas delay time, while keeping all other control parameters at their median position. The simulation's ability to predict changes due to varying the other control parameters is now studied. There are four 'active' parameters, identified by equations (1) and (2): set tool temperature (STT), set melt temperature (SMT), injection velocity (IV), and gas pressure (GP). The previous simulations were made at the median values (i.e. $\mathrm{STT}=\mathrm{SMT}=\mathrm{IV}=\mathrm{GP}=0$ ). The experimental model is now used to predict wall thickness at STT $=1\left(60^{\circ} \mathrm{C}\right.$, from Table 2), and STT $=-1\left(30^{\circ} \mathrm{C}\right)$, and perform the corresponding simulation at a fixed gas delay time of $1.0 \mathrm{~s}$. Similarly, the other three process variables were changed to +1 and -1 , and results compared. For gas pressure, a linear relationship was assumed between pressure and flowrate, and the gas source value was modified accordingly. For each of these eight situations, the predicted change from the experimental model, and the change seen in the simulation were calculated. These are plotted in Fig. 15.

Figure $15 a$ compares results from the experimental model, and the simulation model for the LHS/RHS walls. The experimental model predicts a decrease of $0.10 \mathrm{~mm}$ at $\mathrm{IV}=1$, experiment gives a decrease of $0.08 \mathrm{~mm}$, which can be considered quantitatively correct. For IV $=-1$, the experimental model predicts a rise in wall thickness, of $0.10 \mathrm{~mm}$, compared with $0.03 \mathrm{~mm}$ from the simulation. This suggests a good qualitative agreement from the simulation. The gas pressure (GP) and set melt temperature (STT) variables were not found to be statistically significant, and do not figure in the experimental models; hence the experimental model predicts no change. The simulation model does predict changes, it is perhaps significant that these are of the order of the 'noise' in the experimental model, $0.03 \mathrm{~mm}$. The predictions for changes due to altering the melt temperature, SMT, are again qualitatively correct.

Figure $15 b$ compares results for the fixed/moving walls. Injection velocity, gas pressure, and set tool temperature are in qualitative agreement, the changes are consistently in the correct direction, and quantitative to within a factor of 2 . This suggests that the essentials of the physics of the process are captured within the simulation model. Comparing the magnitudes of the changes with those seen for the LHS/RHS walls, the fixed/moving walls show relatively little change; a possible reason is that the higher average shears in this direction 'mask' the changes (which are predominantly in the temperature field) caused by injection velocity, gas pressure, and set temperatures.

\section{Discussion and conclusions}

The 'design of experiment' approach used to determine the residual wall thickness reduced the number of experiments, and the subsequent cross-sectioning of samples for measuring, to a practical number. The method was able to positively identify which process parameters gave a change that was statistically significant over 180 experiments. The coefficients indicate the relative importance of each process variable as the experimentally derived models are normalised to realistic ranges of each. The models have a particular merit as they permit interpolation to any setting within the range of the process variables; this can be compared directly to the residual wall thicknesses predicted by simulation.

The heat transfer to the mould walls is clearly critical for residual wall thickness. A relationship between residual wall thickness and the penetration depth of cooling from the walls is clear in Fig. 14. In that instance, the depth to which $50 \%$ of the potential temperature change has reached is shown. Two things to be considered are, first the choice of $50 \%$ depth penetration is to obtain a measure of the depth. A higher criterion, such as $90 \%$ of the potential temperature change, would give a different (shallower) depth at a given time. Second, the polymer comes into contact with the mould walls at different points during melt injection, thus the depth penetration process begins before the start of gas delay time. It is clear from both experiment and simulation that the effect of temperature is strong in determining the residual wall thickness, though the difference in physical thicknesses between the LHS/RHS walls and the fixed/moving walls (again shown by both experiment and simulation) shows that the crosssectional aspect ratio is a major factor. In the experiment, closed loop control was used on the coolant water temperature; direct measurement of the tool temperature showed that this method was maintaining the tool at the 'set' temperature $\pm 3^{\circ} \mathrm{C}$. The experimental model shows that the set tool temperature (STT) does not have a strong effect on residual wall thickness, even when varied over $\pm 15^{\circ} \mathrm{C}$. This suggests that the degree of tool temperature control that was used is sufficient for controlling residual wall thickness. More significant variations in tool temperature due to heat flux from the polymer barrel have been seen in other moulds. ${ }^{17}$

The variation of heat flux at the walls was studied for two methods of enforcing the temperature boundary conditions. The first method was that of setting the boundary to the 'set tool temperature' (from the time polymer first contacts), the second approach included imperfect thermal contact between the coolant water and the mould, and included temperature variations within the mould. The method used was a 1-D 
simulation (as opposed to a full 3-D model of the mould, as used in Refs. 17 and 18). Some significant results were found: despite the differences in 'heat transfer coefficient' (infinity compared with $10^{4}-10^{5}$ ), the differences in heat flux were small (approximately $2 \%$ ). It was found that the mould/polymer interface would be within a few degrees Celsius of the water temperature within $0 \cdot 1 \mathrm{~s}$ of contact for the geometry analysed; this is a direct consequence of the much higher conductivity of the steel. Heat flux and mould temperature were both found to be insensitive to the actual value chosen for the water-steel heat transfer coefficient, provided this value was $\geq 10^{3}$. The analysis of depth penetration and the 1-D simulation on the 'coarse' mesh both showed an underprediction of heat loss for the polymer during the first $0.2 \mathrm{~s}$ (approximately), for a $0.2 \mathrm{~mm}$ grid. This difference in heat flux due to mesh size was shown to diminish, almost entirely, after this initial period.

The simulation method of enforcing the temperature of the coolant water at the wall (from first polymer contact) has a number of merits: it allows the natural thermal transport equation to determine heat loss, subject to a single fixed assumption that the polymer/mould interface is at the coolant water temperature. As shown in Fig. 7, this assumption introduces only a very small error in heat flux, in comparison to results that include conduction through the steel, and the steel/water interface. The figure clearly shows that the error is much smaller than that to be expected from finite mesh size. Raised mould temperatures were computed by Polynkin et al., ${ }^{18}$ due to heat flux from a polymer barrel; in situations where the mould inner-wall temperatures are significantly raised, temperature boundary conditions could be set accordingly using localised temperatures from experiment or simulation. The current method uses a novel method of introducing gas, which much more closely replicates the needle injection used in the GAIM process. The key to the method is to apply the method over a 'sphere' of Gauss points which gives a reasonably smooth bubble around the source; enforcing a source within a single element (or within several adjacent elements) resulted in non-physical preference for some directions.

The simulation was able to give quantitative accuracy on some aspects of residual wall prediction, with qualitative accuracy on most. Against gas delay time, the simulation predicted the thickness of the fixed/moving walls with an accuracy of $\pm 2 \%$, up to a 5 s delay. The LHS/RHS walls were underpredicted by around $7 \%$; however, the simulation correctly gave the LHS/RHS walls as thicker, in absolute measurement. The simulation predicted changes that were, at least, qualitatively correct against variation in set tool temperature (STT), set melt temperature (SMT), injection velocity (IV), and gas pressure (GP). The greatest differences seen were for injection velocity $(0.07 \mathrm{~mm})$, and gas pressure $(0.05 \mathrm{~mm})$, all other differences were less than the average residual error from the experimental model $(0.03 \mathrm{~mm})$. The simulation model is clearly capable of realistic prediction, with reservation about the underprediction of the LHS/ RHS thicknesses. The method of using the coolant temperature as the temperature boundary condition represents an 'upper bound' on heat flux from the polymer, a finite heat transfer coefficient could only reduce this. The underprediction clearly has a different cause; the most likely appears to be that the viscoelasticity of molten polymer is omitted from the model. Viscoelasticity would affect the simulation of residual wall thickness in several ways: these include stress relaxation during the gas delay time, and a tendency for the gas bubble to behave as an elastic membrane. Stress relaxation during delay time would affect the (later) flow during gas injection. Elastic tension (which is normally pronounced at high rates in viscoelastic material) seems likely to give preference towards a hemi-spherical gas bubble front (a simple analogy is the spherical shape preferred by a rubber balloon). This would tend to increase the LHS/RHS residual wall thickness, and appears sufficient to account for the difference. This will be the subject of another study.

\section{References}

1. E. Friedrich: U.S. Patent 4,101,617, 1978.

2. E. Haberstroh and H. Weir: Macromol. Mater. Eng., 2000, 284/285, 76-80.

3. F. Ilinca and J.-F. Hetu: Int. J. Numer. Methods Eng., 2002, 53, 2003-2017.

4. R. D. Chien, S.-C. Chen, M. C. Jeng and H.-Y. Yang: Polymer, 1999, 40, 2949-2959.

5. R. E. Khayat, A. Dedouri and L. P. Hebert: J. Non-Newtonian Fluid Mech., 1995, 57, 253-270.

6. C. T. Li and A. I. Iasayev: Polym. Eng. Sci., 2004, 44, 983-991.

7. C.T. Li, J. W. Shin and A. I. Iasayev: Polym. Eng. Sci., 2004, 44, 992-1002.

8. G. A. A. V. Haagh, H. Zuidema, F. N. van de Vosse, G. W. M. Peters and H. E. H. Meijer: Int. Polym. Process., 1997, 12, 207-215.

9. G. A. A. V. Haagh and H. Zuidema: Int. J. Numer. Methods Fluids, 1997, 12, 207.

10. G. A. A. V. Haagh and F. N. Van De Vosse: Int. J. Numer. Methods Fluids, 1998, 28, 1355-1369.

11. G. A. A. V. Haagh, G. W. M. Peters, F. N. van de Vosse and H. E. H. Meijer: Polym. Eng. Sci., 2001, 41, 449-465.

12. Y. Dimakopoulos and J. Tsamopoulos: J. Non-Newtonian Fluid Mech., 2004, 117, 117-139.

13. F. Belblidia, J. F. T. Pittman, A. Polynkin and J. Sienz: Chem. Eng. Sci., 2005, 60, 4953-4956.

14. A. Polynkin, J. F. T. Pittman and J. Sienz: Chem. Eng. Sci., 2004, 59, 2969-2982.

15. A. Polynkin, J. F. T. Pittman and J. Sienz: Chem. Eng. Sci., 2005, 60, 1591-1602.

16. A. Polynkin, J. F. T. Pittman, F. Belblidia and J. Sienz: Proc. 18th Annu. Meet. Polymer Processing Society, Guimares, Portugal, 2002, paper 286.

17. A. Polynkin, J. F. T. Pittman and J. Sienz: Polym. Eng. Sci., 2005, 45, 1049-1058

18. A. Polynkin, J. F. T. Pittman and J. Sienz: Plast. Rubber Compos., 2005, 34, 236-246.

19. 'Plastics - determination of flexural properties', BSI BS EN ISO 178:1997, BS 2782-3:Method 335 A 1993 (ISO 178:1993).

20. D. M. Grove and T. P. Davis: 'Engineering, quality and experimental design'; 1992, Harlow, Essex, UK, Longman Scientific.

21. W. Y. Fowlkes and C. M. Creveling: 'Engineering methods for robust product design: using Taguchi methods in technology and product development', Engineering Process Improvement Series; 1995, Addison-Wesley.

22. Y. A. Cengel: 'Heat transfer: a practical approach', International Edition, ISBN-0-7-115223-7; 1998, McGraw-Hill.

23. J. P. Holman: 'Heat transfer', 8th edn, ISBN 0-07-114320-3; 1997, McGraw-Hill.

24. P. M. Gresho, S. T. Chan, R. L. Lee and C. D. Upson: Int. J. Numer. Methods Fluids, 1984, 4, 557.

25. A. Polynkin, J. F. T. Pittman and J. Sienz: Chem. Eng. Sci., 2004, 59, 2969-2982.

26. L. Johnson, P. Olley and P. D. Coates: Plast. Rubber Compos., 2000, 29, 31-37.

27. J. D. Ferry: 'Viscoelastic properties of polymers'; 1961, John Wiley \& Sons. 